\title{
ON MULTILINEAR SPECTRAL CLUSTER ESTIMATES FOR MANIFOLDS WITH BOUNDARY
}

\author{
Matthew D. Blair, Hart F. Smith, and Christopher D. Sogge \\ AbStRaCt. We prove bilinear and trilinear estimates for the spectral cluster operator \\ on two and three-dimensional compact manifolds with boundary. These are the natural \\ analogs of earlier estimates for the boundaryless case of Burq, Gérard, and Tzvetkov [1], \\ [2]. Our theorem reduces to establishing inequalities over small cubes whose size depends \\ on frequency. After rescaling, these inequalities follow from mixed $L^{p}$ norm estimates \\ on squarefunctions associated to the wave equation.
}

\section{Introduction}

Let $\left(M^{n}, \mathrm{~g}\right)$ be a smooth, compact $n$-dimensional Riemannian manifold with boundary and let $\Delta$ be the corresponding Laplace-Beltrami operator acting on functions. If the boundary is non-empty, we assume that either Dirichlet or Neumann conditions are imposed along $\partial M^{n}$.

Consider the operators $\chi_{\lambda}$ defined as projection onto the subspace spanned by the Dirichlet (or Neumann) eigenfunctions whose corresponding eigenvalues $-\lambda_{j}^{2}$ satisfy $\lambda_{j} \in[\lambda-1, \lambda]$. In the case that $\partial M^{n}$ is empty, it was established in [11] that the following, best possible $L^{2} \rightarrow L^{q}$ estimates hold for $\chi_{\lambda}$ :

$$
\left\|\chi_{\lambda}\right\|_{L^{2} \rightarrow L^{q}} \lesssim \begin{cases}\lambda^{\frac{n-1}{2}\left(\frac{1}{2}-\frac{1}{q}\right)} & 2 \leq q \leq \frac{2(n+1)}{n-1} \\ \lambda^{n\left(\frac{1}{2}-\frac{1}{q}\right)-\frac{1}{2}} & \frac{2(n+1)}{n-1} \leq q \leq \infty\end{cases}
$$

Recently, in [1] and [2], Burq, Gérard, and Tzvetkov established multilinear versions of these estimates, also under the assumption that the boundary of $M$ is empty. To state these, suppose that $\lambda \geq \mu \geq \nu \geq 1$. Then

$$
\begin{gathered}
\left\|\chi_{\lambda} f \chi_{\mu} g\right\|_{L^{2}(M)} \lesssim \Lambda(\mu)\|f\|_{L^{2}(M)}\|g\|_{L^{2}(M)} \\
\left\|\chi_{\lambda} f \chi_{\mu} g \chi_{\nu} h\right\|_{L^{2}(M)} \lesssim(\mu \nu)^{\frac{2 n-3}{4}}\|f\|_{L^{2}(M)}\|g\|_{L^{2}(M)}\|h\|_{L^{2}(M)}
\end{gathered}
$$

where in the first estimate

$$
\Lambda(\mu)= \begin{cases}\mu^{\frac{1}{4}} & n=2 \\ \mu^{\frac{1}{2}}(\log \mu)^{\frac{1}{2}} & n=3 \\ \mu^{\frac{n-2}{2}} & n \geq 4\end{cases}
$$

With the exception of the logarithmic loss for $n=3$, the linear estimate (1.1) with $q=4$ follows as a corollary of the bilinear estimate (1.2), by taking $\lambda=\mu$ and $f=g$. Similarly, the trilinear estimate (1.3) implies (1.1) with $q=6$. Moreover, by taking

Received by the editors March 22, 2007.

The authors were supported by the National Science Foundation grants DMS-0140499, DMS0354668, DMS-0555162, and DMS-0354386. 
$h$ constant and $\nu=1,(1.3)$ implies (1.2) in case $n=2$. For $n \geq 4$, however, the trilinear estimate can be improved by using (1.2) together with the $L^{\infty}$ bounds (1.1) on $h$.

In the case where $\partial M^{n}$ is nonempty, the issue of spectral cluster estimates is considerably more intricate. Here the Rayleigh whispering gallery modes provide examples of spectral clusters which concentrate in a $\lambda^{-\frac{2}{3}} \times \lambda^{-\frac{n-2}{2}}$ neighborhood of a boundary geodesic (see Grieser [4]). These examples show that one cannot achieve linear spectral cluster estimates better than

$$
\left\|\chi_{\lambda}\right\|_{L^{2} \rightarrow L^{q}} \lesssim \begin{cases}\lambda^{\left(\frac{2}{3}+\frac{n-2}{2}\right)\left(\frac{1}{2}-\frac{1}{q}\right)} & 2 \leq q \leq \frac{6 n+4}{3 n-4} \\ \lambda^{n\left(\frac{1}{2}-\frac{1}{q}\right)-\frac{1}{2}} & \frac{6 n+4}{3 n-4} \leq q \leq \infty\end{cases}
$$

The estimates (1.4) were recently proven for dimension $n=2$ in [10], along with partial results in higher dimensions. The question of whether or not they hold in general in higher dimensions remains open. It should be noted that the Rayleigh mode construction can only be done near a portion of the boundary that is strictly convex with respect to the metric g. When the boundary is strictly concave, $\chi_{\lambda}$ actually satisfies (1.1), as was shown by Grieser [4] when $n=2$, and subsequently for all $n$ by [9].

In this work, we establish the following multilinear spectral cluster estimates on a general Riemannian manifold with boundary. We restrict attention to dimensions $n=2,3$, since that is where our results are in some context sharp.

Theorem 1.1. Let $\left(M^{n}, \mathrm{~g}\right)$ and $\chi_{\lambda}$ be as above, with either Dirichlet or Neumann eigenfunctions, and let $\lambda \geq \mu \geq \nu$. Then the following bilinear estimate holds

$$
\left\|\chi_{\lambda} f \chi_{\mu} g\right\|_{L^{2}(M)} \lesssim \Lambda(\mu)\|f\|_{L^{2}(M)}\|g\|_{L^{2}(M)}
$$

with $\Lambda$ defined as

$$
\Lambda(\mu)= \begin{cases}\mu^{\frac{1}{3}} & n=2 \\ \mu^{\frac{2}{3}}(\log \mu)^{\frac{1}{2}} & n=3\end{cases}
$$

In addition, the following trilinear estimate holds for $n=2,3$

$$
\left\|\chi_{\lambda} f \chi_{\mu} g \chi_{\nu} h\right\|_{L^{2}(M)} \lesssim(\mu \nu)^{\frac{3 n-4}{6}}\|f\|_{L^{2}(M)}\|g\|_{L^{2}(M)}\|h\|_{L^{2}(M)}
$$

For $n=2$, the bilinear and trilinear estimates imply the estimate (1.4), respectively for $q=4$ and $q=6$. Moreover, (1.6) implies (1.5) for Neumann conditions by taking $h$ constant. For $n=3$ this is no longer the case. However, the bilinear estimate for $n=3$ implies (up to a logarithmic loss) the best possible $L^{4}$ linear estimate for manifolds with Lipschitz metric, by the examples of [8], and our proof in fact establishes Theorem 1.1 in this context.

As in [10], a key step is to work on the double $\tilde{M}$ of the manifold $M$, obtained by attaching two copies of $M$ along the boundary, and taking coordinate patches along $\partial M \subset \tilde{M}$ which agree with geodesic normal coordinates $\left(x^{\prime}, x_{n}\right)$ on each copy of $M$. In these coordinates, the lift $\tilde{\mathrm{g}}$ of $\mathrm{g}$ to $\tilde{M}$ is given by $\mathrm{g}^{i j}\left(x^{\prime},\left|x_{n}\right|\right)$, and hence g extends to $\tilde{M}$ with a Lipschitz type singularity along $\partial M$. Dirichlet and Neumann eigenfunctions on $M$ correspond to eigenfunctions on $\tilde{M}$ which are, respectively, odd or even under $x_{n} \rightarrow-x_{n}$. Hence, $L^{q}$ bounds on spectral clusters, and the multilinear analogs we consider, can be obtained by proving the same bounds on $(\tilde{M}, \tilde{\mathrm{g}})$. 
The linear estimates of [10] were obtained by establishing mixed-norm $L_{x}^{q} L_{t}^{2}$ estimates on $\tilde{M}$ for the evolution of a spectral cluster under the wave equation, and we follow a similar approach here. In that paper, the precise nature of the singularity of $\tilde{g}$ along $\partial M$ was used, and a microlocal decomposition of the cluster was made in terms of angle from tangent to $\partial M$. Estimates were obtained over small slabs, with size depending on the frequency and angle, and summing over slabs led to a frequency dependent loss for the estimates on $\tilde{M}$.

In contrast, the results of this paper go through generally for the case of a boundaryfree Riemmanian manifold with metric of Lipschitz regularity, as with the linear spectral cluster estimates of [7], or the Strichartz estimates of Tataru [12]. As in those papers, we obtain estimates over small slabs with size depending on the frequency, and use a rescaling argument to reduce matters to obtaining estimates for $C^{2}$ metrics. We then use wave packet methods to obtain dispersive estimates, as in [5], [6] and [12]. Summing over slabs then leads to a frequency dependent loss.

\section{Microlocal Reductions}

For the remainder of this paper, we assume $M$ is a compact manifold without boundary, and $\mathrm{g}$ is a metric of Lipschitz regularity. The condition that $f, g, h$ be spectrally localized can be relaxed, and we work instead with a quasimode condition. We state the condition for $f$ here, the condition for $g$ and $h$ being analogous. For each local coordinate chart we write

$$
\mathrm{g} d^{2} f+\lambda^{2} f=w, \quad \mathrm{~g} d^{2}=\sum_{j, k} \mathrm{~g}^{j k}(x) \partial_{j} \partial_{k} .
$$

Given such an equation, we set

$$
\text { | }|f|\left\|_{\lambda}=\right\| f\left\|_{L^{2}}+\lambda^{-1}\right\| d f\left\|_{L^{2}}+\lambda^{-2}\right\| d^{2} f\left\|_{L^{2}}+\lambda^{-1}\right\| w \|_{L^{2}} .
$$

If $f=\chi_{\lambda} f$, then for $\phi$ a smooth cutoff to a local coordinate system, the function $\phi f$ satisfies the equation (2.1) on $\mathbb{R}^{n}$, and

$$
\|\phi f\|_{\lambda} \lesssim\|f\|_{L^{2}(M)} .
$$

For the bilinear estimates it thus suffices to prove, for each coordinate chart, that

$$
\|\phi f \phi g\|_{L^{2}} \lesssim \Lambda(\mu)\|\mid \phi f\|_{\lambda}\|\| \phi g \|_{\mu},
$$

and analogously for the trilinear version.

By choosing appropriate coordinates, we may assume $f$ satisfies $(2.1)$ on $\mathbb{R}^{n}$, with $f$ supported in the unit ball, and

$$
\left\|\mathrm{g}^{i j}-\delta^{i j}\right\|_{\operatorname{Lip}\left(\mathbb{R}^{n}\right)} \leq c_{0},
$$

with $c_{0}$ a constant to be chosen suitably small.

Let $S_{r}=S_{r}(D)$ denote a smooth cutoff on the Fourier transform side to frequencies of size $|\xi| \leq r$. Let $\mathrm{g}_{\lambda}=S_{c^{2} \lambda} \mathrm{g}$, for $c$ to be chosen suitably small. Then

$$
\left\|\left(\mathrm{g}-\mathrm{g}_{\lambda}\right) d^{2} f\right\|_{L^{2}} \lesssim c^{-2} \lambda^{-1}\left\|d^{2} f\right\|_{L^{2}},
$$

and thus we may replace $\mathrm{g}$ by $\mathrm{g}_{\lambda}$ in (2.1) at the expense of absorbing the above term into $w$, which does not change the size of $\||f|\|_{\lambda}$. 
We next take a microlocal partition of unity, $1=\sum \Gamma(D)$, where each $\Gamma(\xi)$ is a smooth symbol of order 0 supported in a cone of small angle. By the Coifman-Meyer commutator theorem [3], since $\mathrm{g}$ is Lipschitz

$$
[\mathrm{g}, \Gamma(D)] d: L^{2}\left(\mathbb{R}^{n}\right) \rightarrow L^{2}\left(\mathbb{R}^{n}\right),
$$

hence $\Gamma(D) f$ satisfies the equation (2.1), with ||$|\Gamma(D) f|\left\|\left.\right|_{\lambda} \lesssim\right\||| f \mid \|_{\lambda}$.

Since there are finitely many terms, we may replace $f$ by $\Gamma(D) f$, which is no longer compactly supported, but is rapidly decreasing and smooth outside the unit ball. Without loss of generality we assume that $\Gamma(\xi)$ is supported within a small angle of the $\xi_{1}$ axis. We similarly replace $g$ and $h$ by $\Gamma^{\prime}(D) g$ and $\Gamma^{\prime \prime}(D) h$, localized in frequency to small cones along general directions.

Letting $x^{\prime}=x_{2}$ in case of dimension $n=2$, and $x^{\prime}=\left(x_{2}, x_{3}\right)$ in case of dimension $n=3$, we bound

$$
\begin{aligned}
\|f g\|_{L^{2}} & \leq\|f\|_{L_{x_{1}}^{\infty} L_{x^{\prime}}^{2}}\|g\|_{L_{x_{1}}^{2} L_{x^{\prime}}^{\infty}} \\
\|f g h\|_{L^{2}} & \leq\|f\|_{L_{x_{1}}^{\infty} L_{x^{\prime}}^{2}}\|g\|_{L_{x_{1}}^{4} L_{x^{\prime}}^{\infty}}\|h\|_{L_{x_{1}}^{4} L_{x^{\prime}}^{\infty}}^{\infty}
\end{aligned}
$$

Since $\Gamma(D) f$ is rapidly decreasing outside the unit ball, it suffices to take the norms above over the ball of radius 2. Theorem 1.1 is then a result of the following.

Theorem 2.1. Suppose that $f$ satisfies the equation

$$
\mathrm{g}_{\lambda} d^{2} f+\lambda^{2} f=w
$$

Then the following hold, where the norms on the left side are over a bounded set

$$
\begin{aligned}
& \|f\|_{L_{x_{1}}^{2} L_{x^{\prime}}^{\infty}} \lesssim \lambda^{\frac{2}{3}}(\log \lambda)^{\frac{1}{2}}\|f \mid\|_{\lambda}, \quad n=3 \\
& \|f\|_{L_{x_{1}}^{4} L_{x^{\prime}}^{\infty}} \lesssim \begin{cases}\lambda^{\frac{1}{3}}|||f| \|_{\lambda}, & n=2 \\
\lambda^{\frac{5}{6}}|||f| \|_{\lambda}, & n=3\end{cases}
\end{aligned}
$$

Futhermore, if $\hat{f}(\xi)$ is supported in a small cone about the $\xi_{1}$ axis, then

$$
\|f\|_{L_{x_{1} L_{x^{\prime}}}^{2}} \lesssim\||| f \mid\|_{\lambda}
$$

Proof. We start by localizing $f$ dyadically in frequency. Let

$$
f=S_{c \lambda} f+\left(S_{c^{-1} \lambda}-S_{c \lambda}\right) f+\left(1-S_{c^{-1} \lambda}\right) f \equiv f_{<\lambda}+f_{\lambda}+f_{>\lambda} .
$$

Since $\left[S_{c \lambda}, \mathrm{g}_{\lambda}\right] d: L^{2} \rightarrow L^{2}, f_{\lambda}$ satisfies (2.2), with $\left\|\left|w_{\lambda}\right|||_{\lambda} \lesssim\right\|\|f \mid\|_{\lambda}$, and similarly for $f_{>\lambda}$ and $f_{<\lambda}$. Furthermore, by the frequency localization of $g_{\lambda}$, each of $w_{\lambda}, w_{<\lambda}$, and $w_{>\lambda}$ is also localized to the appropriate range of frequencies.

A simple integration by parts argument (see the proof of Corollary 5 of [7]) yields that, for $c$ sufficiently small,

$$
\lambda\left\|f_{<\lambda}\right\|_{L^{2}\left(\mathbb{R}^{n}\right)}+\left\|d f_{>\lambda}\right\|_{L^{2}\left(\mathbb{R}^{n}\right)} \lesssim\|\| f \|_{\lambda} .
$$

Elliptic regularity additionally gives the bound

$$
\left\|d^{2} f_{>\lambda}\right\|_{L^{2}\left(\mathbb{R}^{n}\right)} \lesssim \lambda\||| f\|_{\lambda} .
$$


Sobolev embedding then yields each of the estimates (2.3)-(2.5) for $f_{<\lambda}$ and $f_{>\lambda}$. Indeed, there is a gain of $\lambda^{\frac{2}{3}}(\log \lambda)^{\frac{1}{2}}$ in the estimate (2.3), and a gain of $\lambda^{\frac{7}{12}}$ in the estimate (2.4), for these terms.

Consequently, we are reduced to establishing $(2.3)-(2.5)$ for the term $f_{\lambda}$. We start with (2.5). Let $V$ denote the vector field

$$
V=2\left(\partial_{1} f_{\lambda}\right) g_{\lambda} d f_{\lambda}+\left(\lambda^{2} f_{\lambda}^{2}-\left\langle g_{\lambda} d f_{\lambda}, d f_{\lambda}\right\rangle\right) \overrightarrow{e_{1}}
$$

Then

$$
\operatorname{div} V=2\left(\partial_{1} f_{\lambda}\right)\left(\operatorname{div} g_{\lambda}\right) \cdot d f_{\lambda}+2\left(\partial_{1} f_{\lambda}\right) w_{\lambda}-\left\langle\left(\partial_{1} g_{\lambda}\right) d f_{\lambda}, d f_{\lambda}\right\rangle .
$$

Applying the divergence theorem on the set $x_{1} \leq r$ yields

$$
\int_{x_{1}=r} V_{1} d x^{\prime} \lesssim \lambda^{2}\left\|f_{\lambda}\right\|_{L^{2}\left(\mathbb{R}^{n}\right)}^{2}+\left\|d f_{\lambda}\right\|_{L^{2}\left(\mathbb{R}^{n}\right)}^{2}+\left\|w_{\lambda}\right\|_{L^{2}\left(\mathbb{R}^{n}\right)}^{2} .
$$

Since $g_{\lambda}$ is pointwise close to the flat metric, we have pointwise that

$$
V_{1} \geq \frac{3}{4}\left|\partial_{1} f_{\lambda}\right|^{2}+\frac{3}{4} \lambda^{2}\left|f_{\lambda}\right|^{2}-\left|\partial_{x^{\prime}} f_{\lambda}\right|^{2} .
$$

The frequency localization of $\widehat{f}_{\lambda}$ to $\left|\xi^{\prime}\right| \leq c \lambda$ yields

$$
\int_{x_{1}=r} V_{1} d x^{\prime} \geq \frac{1}{2} \int_{x_{1}=r}\left|d f_{\lambda}\right|^{2}+\lambda^{2}\left|f_{\lambda}\right|^{2} d x^{\prime} .
$$

Consequently,

$$
\begin{aligned}
\lambda^{-1}\left\|d f_{\lambda}\right\|_{L_{x_{1}}^{\infty} L_{x^{\prime}}^{2}}+\left\|f_{\lambda}\right\|_{L_{x_{1}}^{\infty} L_{x^{\prime}}^{2}} & \lesssim\left\|f_{\lambda}\right\|_{L^{2}\left(\mathbb{R}^{n}\right)}+\lambda^{-1}\left\|d f_{\lambda}\right\|_{L^{2}\left(\mathbb{R}^{n}\right)}+\lambda^{-1}\left\|w_{\lambda}\right\|_{L^{2}\left(\mathbb{R}^{n}\right)} \\
& \leq\left\|f_{\lambda}\right\| \|_{\lambda},
\end{aligned}
$$

yielding estimate (2.5).

For the remainder, we assume that $\hat{f}(\xi)$ is localized to a small cone along the direction $\omega$. In this case, the above argument yields uniform $L^{2}$ bounds over hyperplanes of the form $\omega \cdot x=r$. In the proof of (2.3)-(2.4) we will use the following consequence. Suppose that $S_{R}$ is a slab of the form $\omega \cdot x \in I$, where $I$ is an interval of length $|I|=R$. Then

$$
\lambda^{-1}\left\|d f_{\lambda}\right\|_{L^{2}\left(S_{R}\right)}+\left\|f_{\lambda}\right\|_{L^{2}\left(S_{R}\right)} \lesssim R^{\frac{1}{2}}\|\| f_{\lambda} \|_{\lambda} .
$$

We cover the bounded set on which the norms in (2.3) and (2.4) are taken by $\approx \lambda^{\frac{1}{3}}$ slabs of the form $\omega \cdot x \in I$, where $|I|=R=\lambda^{-\frac{1}{3}}$. Then

$$
\left\|f_{\lambda}\right\|_{L_{x_{1}}^{p} L_{x^{\prime}}^{\infty}} \lesssim \lambda^{\frac{1}{3 p}} \sup _{S_{R}}\left\|f_{\lambda}\right\|_{L_{x_{1}}^{p} L_{x^{\prime}}^{\infty}\left(S_{R}\right)} .
$$

We will establish the following result. Suppose that $Q_{R}$ is a cube of sidelength $R=$ $\lambda^{-\frac{1}{3}}$, and $Q_{R}^{*}$ its double. Then

$$
\begin{aligned}
\left\|f_{\lambda}\right\|_{L_{x_{1}}^{p} L_{x^{\prime}}^{\infty}\left(Q_{R}\right)} & \\
& \lesssim c_{p}(\lambda) R^{-\frac{1}{2}}\left(\left\|f_{\lambda}\right\|_{L^{2}\left(Q_{R}^{*}\right)}+\lambda^{-1}\left\|d f_{\lambda}\right\|_{L^{2}\left(Q_{R}^{*}\right)}+R \lambda^{-1}\left\|w_{\lambda}\right\|_{L^{2}\left(Q_{R}^{*}\right)}\right)
\end{aligned}
$$

where

$$
c_{2}(\lambda)=\lambda^{\frac{1}{2}}(\log \lambda)^{\frac{1}{2}}, \quad n=3 \quad c_{4}(\lambda)= \begin{cases}\lambda^{\frac{1}{4}}, & n=2 \\ \lambda^{\frac{3}{4}}, & n=3\end{cases}
$$


If we cover the slab $S_{R}$ by disjoint cubes $Q_{R}$, then by (2.6) we obtain

$$
\left\|f_{\lambda}\right\|_{L_{x_{1}}^{p} L_{x^{\prime}}^{\infty}\left(S_{R}\right)} \lesssim c_{p}(\lambda)\|\| f_{\lambda}\|\|_{\lambda}
$$

and (2.7) yields (2.3)-(2.4).

The estimate (2.8) is scale-invariant. Precisely, if we change $x \rightarrow R x$, so that $Q$ becomes a cube of size 1 , and $f_{\lambda}(R \cdot)$ is frequency localized at scale $R \lambda=\lambda^{\frac{2}{3}}$, then, with $\mu=\lambda^{\frac{2}{3}}$, estimate $(2.8)$ is equivalent to the following

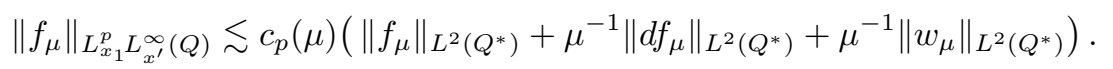

Here, $f_{\mu}(x)=f_{\lambda}\left(\lambda^{-\frac{1}{3}} x\right)$, which satisfies the equation

$$
\mathrm{g}_{\mu} d^{2} f_{\mu}+\mu^{2} f_{\mu}=w_{\mu},
$$

with $\mathrm{g}_{\mu}(x)=\mathrm{g}_{\lambda}\left(\lambda^{-\frac{1}{3}} x\right)$. Observe that

$$
\left\|d \mathrm{~g}_{\mu}\right\|_{L^{\infty}} \leq c_{0} \lambda^{-\frac{1}{3}}=c_{0} \mu^{-\frac{1}{2}}
$$

hence

$$
\left\|\mathrm{g}_{\mu}-\mathrm{g}_{\mu^{1 / 2}}\right\|_{L^{\infty}} \leq c_{0} \mu^{-1}
$$

with $\mathrm{g}_{\mu^{1 / 2}}=S_{c^{2} \mu^{1 / 2}} \mathrm{~g}_{\mu}$. Thus $f_{\mu}$ satisfies the equation

$$
\mathrm{g}_{\mu^{1 / 2}} d^{2} f_{\mu}+\mu^{2} f_{\mu}=w_{\mu},
$$

with the right-hand side of (2.9) of comparable size.

It follows from (2.10) that the metric $\mathrm{g}_{\mu^{1 / 2}}$ is of regularity $C^{2}$. Indeed,

$$
\left\|\mathrm{g}_{\mu^{1 / 2}}^{j k}-\delta^{j k}\right\|_{C^{2}} \leq c_{0} .
$$

To establish (2.9), we may thus use techniques developed to establish dispersive estimates for operators of principal type with $C^{2}$ coefficients. We follow below the path through squarefunction estimates for solutions to a first order hyperbolic equation, by introducing a time variable, as in [6]. It should also be possible to establish the dispersive estimates directly for (2.11) using methods of [5].

Let

$$
p(\cdot, \xi)=S_{c^{2} \mu^{1 / 2}}\left(\sum_{j, k} \mathrm{~g}_{\mu^{1 / 2}}^{j k}(\cdot) \xi_{j} \xi_{k}\right)^{\frac{1}{2}} .
$$

Then

$$
\left\|p(x, D)^{2} f_{\mu}+\mathrm{g}_{\mu^{1 / 2}} d^{2} f_{\mu}\right\|_{L^{2}\left(\mathbb{R}^{n}\right)} \lesssim \mu\left\|f_{\mu}\right\|_{L^{2}}
$$

Thus,

$$
(\mu+p(x, D))(\mu-p(x, D)) f_{\mu}=w_{\mu},
$$

with the error harmlessly absorbed into $w_{\mu}$. The operator $\mu+p(x, D)$ is elliptic on the frequency support of $(\mu-p(x, D)) f_{\mu}$, hence we may write

$$
(\mu-p(x, D)) f_{\mu}=\mu^{-1} w_{\mu},
$$

with $\mid\|f\|_{\lambda}$ still of comparable size. Finally, let

$$
u(t, x)=e^{-i t \mu} f_{\mu}(x), \quad F=\mu^{-1} e^{-i t \mu} w_{\mu} .
$$

Then

$$
\left(\partial_{t}+i p(x, D)\right) u=F
$$


and it suffices to show that

$$
\|u\|_{L_{x_{1}}^{p} L_{x^{\prime}}^{\infty} L_{t}^{2}(Q \times[0,1])} \lesssim c_{p}(\mu)\left(\|u\|_{L_{t}^{\infty} L_{x}^{2}\left([0,1] \times \mathbb{R}^{n}\right)}+\|F\|_{L_{t}^{1} L_{x}^{2}\left([0,1] \times \mathbb{R}^{n}\right)}\right) .
$$

Our proof of (2.12) follows very closely the proof of the linear spectral cluster estimates in [6]; we outline just the main steps here. Following [6, §3], consider the wave packet transform of $u_{\mu}$,

$$
\left(T_{\mu} u\right)(t, x, \xi)=\mu^{\frac{n}{4}} \int e^{-i\langle\xi, z-x\rangle} \phi\left(\mu^{\frac{1}{2}}(z-x)\right) u(t, z) d z,
$$

where $\phi$ is a real, even Schwartz function, with $\|\phi\|_{L^{2}}=(2 \pi)^{-\frac{n}{2}}$, and with Fourier transform supported in the unit ball $\{|\xi| \leq 1\}$. Then

$$
\partial_{t} T_{\mu} u(t, x, \xi)=\left(d_{\xi} p(x, \xi) \cdot d_{x}-d_{x} p(x, \xi) \cdot d_{\xi}\right) T_{\mu} u(t, x, \xi)+G(t, x, \xi),
$$

where $G(t, x, \xi)=0$ unless $\frac{1}{8} \mu<|\xi|<2 \mu$, and

$$
\|G\|_{L_{t}^{1} L_{x, \xi}^{2}} \lesssim\|u\|_{L_{t}^{1} L_{x}^{2}}+\|F\|_{L_{t}^{1} L_{x}^{2}}
$$

Let $\chi_{t}$ denote the canonical transform on $\mathbb{R}_{x, \xi}^{2 n}=T^{*}\left(\mathbb{R}^{n}\right)$ generated by the Hamiltonian flow of $p$. Thus, $\chi_{t}(x, \xi)=\gamma(t)$, where $\gamma$ is the integral curve with $\gamma(0)=(x, \xi)$. Then we have

$$
\left(T_{\mu} u\right)(t, x, \xi)=\left(T_{\mu} u\right)\left(0, \chi_{-t}(x, \xi)\right)+\int_{0}^{t} G\left(r, \chi_{r-t}(x, \xi)\right) d r .
$$

Thus, $T_{\mu} u(t, x, \xi)$ is an integrable superposition over $r$ of $1_{t>r}$ multiplied by a function invariant under the Hamiltonian flow of $p$.

Since $u(t, x)=T_{\mu}^{*}\left(T_{\mu} u\right)(t, x, \xi)$, it suffices to show

$$
\|W \tilde{f}\|_{L_{x}^{p} L_{x^{\prime}}^{\infty} L_{t}^{2}\left(\mathbb{R}^{n} \times[0,1]\right)} \lesssim c_{p}(\mu)\|f\|_{L_{x, \xi}^{2}},
$$

where

$$
(W \tilde{f})(t, x)=T_{\mu}^{*}\left(\tilde{f} \circ \chi_{-t}\right)(x) .
$$

This is in turn equivalent to the following bounds

$$
\left\|W W^{*} F\right\|_{L_{x_{1}}^{p} L_{x^{\prime}}^{\infty} L_{t}^{2}(Q \times[0,1])} \lesssim c_{p}(\mu)^{2}\|F\|_{L_{x_{1}}^{p^{\prime} L_{x^{\prime}}^{1} L_{t}^{2}(Q \times[0,1])}} .
$$

The operator $W W^{*}$ has an integral kernel $K$ which is highly localized to a $\mu^{-1}$ neighborhood of the light cone, with the dispersive rate of decay away from the origin, see $[6,(3.11)]$,

$$
|K(s, y ; t, z)| \lesssim \mu^{n}\left(1+\mu\left|y_{1}-z_{1}\right|\right)^{-\frac{n-1}{2}}(1+\mu|d(y, z)-| s-t||)^{-N}
$$

with $d(y, z)$ the distance of $y$ to $z$ determined by $p$.

We remark that in [6] this estimate was established assuming the kernel was microlocalized near the $\xi_{1}$ axis. That assumption, however, was necessary for $L^{2}$-energy estimates, not the above dispersive estimates. Indeed, the proof of $[6,(3.11)]$ establishes (2.15) with $\left|y_{1}-z_{1}\right|$ replaced by $|y-z|$, since $|t-s| \approx|y-z|$ on the light cone, and hence holds without any assumption of conic micro-localization.

Estimate (2.15) implies that, for each $\left(y_{1}, z_{1}\right)$,

$$
\left\|\int K(s, y ; t, z) v\left(t, z^{\prime}\right) d t d z^{\prime}\right\|_{L_{y^{\prime}}^{\infty} L_{s}^{2}} \lesssim \mu^{n-1}\left(1+\mu\left|y_{1}-z_{1}\right|\right)^{-\frac{n-1}{2}}\|v\|_{L_{z^{\prime}}^{1} L_{t}^{2}} .
$$


For $n=3$ and $p=2$, estimate (2.14) follows from

$$
\int_{\left|z_{1}\right| \leq 2} \mu^{2}\left(1+\mu\left|y_{1}-z_{1}\right|\right)^{-1} \leq \mu \log \mu .
$$

For $p=4,(2.14)$ follows from the Hardy-Litlewood-Sobolev inequality, together with the bound

$$
\mu^{n-1}\left(1+\mu\left|y_{1}-z_{1}\right|\right)^{-\frac{n-1}{2}} \leq \mu^{n-\frac{3}{2}}\left|y_{1}-z_{1}\right|^{-\frac{1}{2}} .
$$

\section{References}

[1] Burq, N., Gérard, P. and Tzvetkov, N., Bilinear eigenfunction estimates and the nonlinear Schrödinger equation on surfaces. Invent. Math. 159 (2005), 187-223.

[2] _ Multilinear eigenfunction estimates for the Laplace spectral projectors on compact manifolds. C. R. Math. Acad. Sci. Paris 338 (2004), no. 5, 359-364.

[3] Coifman, R. and Meyer, Y., Commutateurs d'integrales singulieres et operateurs multilineaires. Ann. Inst. Fourier Grenoble 28 (1978), 177-202.

[4] Grieser, D., $L^{p}$ bounds for eigenfunctions and spectral projections of the Laplacian near concave boundaries. Thesis, UCLA, 1992.

[5] Koch, H.; Tataru, D. Dispersive estimates for principally normal operators. Comm. Pure Appl. Math 58 (2005), 217-284.

[6] Smith, H.F., Spectral cluster estimates for $C^{1,1}$ metrics. Amer. J. Math. 128 (2006), 1069-1103.

[7] Sharp $L^{2} \rightarrow L^{q}$ bounds on spectral projectors for low regularity metrics. Math. Res. Lett. 13 (2006), no. 6, 965-972.

[8] Smith, H.F. and Sogge, C.D., On Strichartz and eigenfunction estimates for low regularity metrics. Math. Res. Lett. 1 (1994), 729-737.

[9] On the critical semilinear wave equation outside convex obstacles. J. Amer. Math. Soc. 8 (1995), 879-916.

[10] On the $L^{p}$ norm of spectral clusters for compact manifolds with boundary. Acta Math. 198 (2007), 107-153.

[11] Sogge, C.D., Concerning the $L^{p}$ norm of spectral clusters for second-order elliptic operators on compact manifolds. J. Funct. Anal. 77 (1988), no. 1, 123-138.

[12] D. Tataru, Strichartz estimates for operators with nonsmooth coefficients III. J. Amer. Math. Soc. 15 (2002), 419-442.

Department of Mathematics, University of Rochester, Rochester, NY 14627

E-mail address: blair@math.rochester.edu

Department of Mathematics, University of Washington, Seattle, WA 98195

E-mail address: hart@math.washington.edu

Department of Mathematics, Johns Hopkins University, Baltimore, MD 21218

E-mail address: sogge@jhu.edu 